\title{
Estudo da demanda ambulatorial da clínica de odontologia da Universidade do Estado do Amazonas
}

\author{
The study of de ambulatory demand of de dental clinic \\ of State University of Amazonas
}

Emílio Carlos Sponchiado Júnior ${ }^{1}$

Torricelly Barreto deSouza ${ }^{2}$

${ }^{1}$ Universidade Federal do Amazonas. Av. Ayrão 1.133, Praça 14. 69000-000

M anaus AM .

spemilio@ig.com.br

${ }^{2}$ Universidade do Estado do

Amazonas.
Abstract Theobjective of this study was to evaluate the demand of the clinic of Dentistry of UEA. One hundred patients who were being treated in the discipline of Semiology answered a questionnaire that collected data on demographic and socio-economic profile. The clinical examination showed the nosologic profile, the blood pressure and dental losses profile. Results showed that 52\% of patients were female, aged between 20 and 29 years, $48.3 \%$ with theincomplete high school and $60.8 \%$ with monthly income greater than five minimum wages. As for dental loss, the teeth most affected were the first molars, while $29 \%$ have lost the top first molar and $45 \%$ the bottom. The prevalent nosologic profile was $31.3 \%$ for treatment in the area of Restorative Dentistry followed by $21 \%$ of Periodontics and 19\% of Surgery. Only one patient had hypertension. Our conclusion is that most of the population that participated on this study was middle-class women with low average level of education and dental precarioustableshowing many dental losses and requiring more rehabilitation treatments than preventive. With a greater understanding of the reality of the users of the UEA dental clinic it will bepossibleto improve the planning of care and actions to improve the promotion of health.

Key words Ambulatory demand, Nosologic and socio economic profile
Resumo 0 objetivo deste trabalho foi avaliar a demanda ambulatorial da Clínica de Odontologia da Universidade do Estado do Amazonas (UEA). Foram entrevistados cem pacientes em tratamento na disciplina de semiologia; por meio de um questionário, foram coletados os dados demográficos e 0 perfil socioeconômico e por meio de exame clínico foram obtidoso perfil nosológico eo perfil da pressão arterial e das perdas dentais. Os resultados evidenciaram que $52 \%$ dospacientes eram do sexo feminino, com idade predominante entre 20 e 29 anos; $48,3 \%$ com o segundo grau incompleto e $60,8 \%$ com renda mensal maior que cinco salários mínimos. Quanto à perda dental, os dentes mais acometidos foram os primeiros molares; $29 \%$ perderam o primeiro molar superior e 45\%, o inferior. 0 perfil nosológico mais encontrado foi de 31,3\% para tratamentos na área de dentística restauradora, seguida de $21 \%$ deperiodontia e $19 \%$ decirurgia. Apenas um paciente apresentava hipertensão. Concluí-se que a população predominantemente atendida foi demulheres declassemédia baixa, com nível médio de instrução e quadro odontológico precário, evidenciando muitas perdas dentais e necessitando mais de tratamentos reabilitadores do que os preventivos. Com a maior compreensão da realidade dos usuários da dínica odontológica da UEA, será possível melhorar o planejamento do atendimento e das ações para promoção de saúde. Palavras-chave Demanda ambulatorial, Perfil nosológico e socioeconômico 


\section{Introdução}

Há um interesse crescente no conhecimento do perfil epidemiológico para a adequação das práticas de saúde. Portanto, conhecer a demanda ambulatorial na rede pública tornou-se tarefa necessária tanto para avaliação de serviços como para orientação dos que trabalham com gerência, programação e planejamento em saúde ${ }^{1}$.

Além disso, as análises de mortalidade, já bastante difundidas, que tradicionalmente subsidiam o planejamento em saúde, devem ser completadas pela análise da morbidade, pois há um grande número de doenças e agravos a saúde que não causam óbito, mas levam a graus variados de espoliação como, por exemplo, as doenças relacionadas com a odontologia, como a cárie e a doença periodontal ${ }^{2}$.

Osestudos de demanda ambulatorial servem basicamente a quatro objetivos: avaliar a qualidade dos registros clínicos, verificar a adesão ao serviço, avaliar o próprio serviço e conhecer as particularidades dos usuários do serviço, podendo assim melhorar suas condições e conhecer a real necessidade da população atendida. O utros aspectos a serem considerados queatingem diretamente os usuários do sistema público de saúde são as transformações demográficas, sociais e econômicas pelas quais passa a sociedade brasileira, e estas impactam diretamente nas condições de vida e saúde da população, ao mesmo tempo em que geram novas demandas para 0 sistema de saúde do país ${ }^{3}$.

Por meio dos estudos de demanda ambulatorial, é possível também monitorar e pré-diagnosticar patologias como a hipertensão, diabetes, cárie, doença periodontal, dentre outras. A saúde bucal em nosso país mantém-se como fator de preocupação, tanto para os profissionais da área quanto para o governo, uma vez que uma minoria da população brasileira possui condições econômicas para arcar com os custos de um atendimento odontológico. Sendo assim, os dirigentes do serviço público deveriam conhecer a população usuária dos serviços para poder aperfeiçoar 0 atendimento, melhorando a qualidade de vida da população ${ }^{1,4}$.

0 presente estudo tem como objetivo analisar a demanda ambulatorial dos usuários da clínica de odontologia da UEA durante 0 ano de 2006, observando o perfil socioeconômico, perfil nosológico e clínico (perdas dentais e pressão arterial).

\section{M ateriais emétodos}

Para constituição da amostra, foi estabelecido um número de cem pacientes examinados por ordem de chegada para atendimento na clínica de odontologia, que se dá pela disciplina de semiologia odontológica. As coletas realizaram-se após triagem semiológica e não houve exclusão de fichas.

Para coleta dos dados mediante as informações dadas pelo sujeito da pesquisa, todos os participantes ou responsáveis assinaram um termo de consentimento livre e esclarecido; a pesquisa foi aprovada pelo CEP/UFAM e o pesquisador utilizou um formulário para a coleta de dados que abordou itens como perfil demográfico, perfil socioeconômico, perfil nosológico, perfil da perda de elementos dentais e da pressão arterial.

$\mathrm{Na}$ avaliação do perfil demográfico e socioeconômico, os dados foram coletados por meio da entrevista realizada entre o pesquisador e 0 sujeito da pesquisa. Já o perfil nosológico e clínico foi definido por meio deexameclínico efísico realizado pelo pesquisador.

Q uanto à análise da perda de el ementos dentais, esta foi realizada colocando o paciente em uma cadeira odontológica e o pesquisador inspecionou a cavidade bucal do pacientecom auxílio de um espelho clínico, indicando o número e localização dos elementos dentais perdidos e a necessidade de tratamento (perfil nosológico). Para mensuração da pressão arterial, foi utilizado o mesmo equipamento e a mesma técnica, um esfignomanômetro de coluna de mercúrio (Premium ${ }^{\circledR}$ ), de fabricação brasileira, e um estetoscópio com diafragma plano da mesma marca. Todas as aferições, assim como as calibrações do aparelho, seguiram rigorosamente as recomendações expressas pela literatura ${ }^{5}$. 0 s pacientes foram classificados de acordo com a Organização Mundial de Saúde (OMS), em normais, normais limítrofes, hipertensão leve e hipotensão. Os dados foram tabulados e analisados pela estatística descritiva no programa M icrosoft O fficeExcel 2007 尺.

\section{Resultados}

Quanto à prevalência dos usuários e sua faixa etária por idade e sexo, os resultados mostraram que $48 \%$ dos pacientes são do sexo masculino e $52 \%$, do sexo feminino. A idade prevalente foi $36 \%$ dos usuários do sexo masculino e $31 \%$ do feminino de 20 a 29 anos, seguida de $20 \%$ do sexo 
masculino e $28 \%$ do feminino de 30 a 39 anos e, respectivamente, 18 e $17 \%$ de pacientes entre $15 \mathrm{e}$ 19 anos, $16 \%$ e $3 \%$ entre 40 e 49 anos, $8 \%$ e $5 \%$ entre 50 e 49 anos e, por fim, somente do sexo feminino, 3\% de pacientes entre 5 a 9 anos, 3\% entre 70 e 79 anos e $2 \%$ com mais de 80 anos.

Os resultados do perfil socioeconômico evidenciaram que $47 \%$ dos usuários possuíam 0 ensino médio completo, $23 \%$, o ensino médio incompleto, $16 \%$, o ensino fundamental completo, $10 \%$, o ensino fundamental incompleto e $4 \%, 0$ ensino superior completo. Quanto à renda mensal, $60 \%$ dos participantes declararam ter uma renda mensal de $R \$ 1.000,00$ a $2.000,00,30 \%$ de $R \$ 500,00$ a $1.000,00,7 \%$ mais que $2.000,00$ reais e $3 \%$ até 500,00 reais.

Os resultados de maior prevalência no perfil nosológico mostraram que $31 \%$ necessitavam de atenção na área de dentística restauradora, 22 \% em periodontia, $20 \%$ em cirurgia, 14 em endodontia e $13 \%$ em próteses.

Dos cem pacientes entrevistados, foi obtida uma variável de 319 perdas dentais para os dentes superiores; 240 para os dentes inferiores com prevalência de molares em ambas as arcadas, sendo o primeiro molar inferior o com maior índice de extração(45\%) seguido do primeiro molar superior $(29 \%)$.

A pós a aferição da pressão arterial, $77 \%$ dos usuários apresentaram níveis de pressão arterial dentro dos valores normais estabelecidos pela Organização M undial deSaúde(OM S), 20\% apresentaram níveis normais limítrofes, $2 \%$ apresentaram uma leve tendência à hipotensão e apenas $1 \%$ desses pacientes apresentaram hipertensão.

\section{Discussão}

A sociedade brasileira vive uma transformação tanto demográfica quanto socioeconômica, impactada pelas condições de vida e saúde da população. A saúde bucal em nosso país e no estado do Amazonas mantém-se como fator de pre ocupação, tanto para os profissionais da área quanto para o governo, uma vez que uma minoria dessa população não possui condiç̧ões econômicas para arcar com os custos de um atendimento odontológico em consultório particular ${ }^{6}$.

O nível socioeconômico, condições culturais, hábitos alimentares eo grau de desenvolvimento global de cada país exercem um papel bem mais representativo na determinação do nível de saúde de uma comunidade. A situação dramática dos países em desenvolvimento, os quais segui- ram os modelos de prestação de serviços odontológicos e de formação de recursos humanos das nações industrializadas, levou à ocorrência deíndices crescentes de doenças bucais. 0 principal desafio a ser vencido pela profissão, nos dias atuais, é a construção de uma odontologia com conteúdo social, dirigida a todos $s^{4,7,8}$.

Frazão et al. ${ }^{3}$, em um estudo sobre as condições de saúde bucal em adultos no estado de São Paulo, afirmaram haver relação entre os indicadores socioeconômicos que mostraram correlação com a proporção de adultos com ao menos vinte dentes funcionais, sendo esses encontrados principalmente em adultos mais velhos, negros, com renda inferior a três salários mínimos que trabal havam em áreas rurais, em cidades pequenas e áreas não fluoretadas. Os resultados obtidos por Frazão et al. ${ }^{3}$ no estado de São Paulo são semelhantes aos deste estudo, sendo que se torna evidente que, dentre os pacientes entrevistados, encontra-se uma pequena parte da população carente, visto que a renda mensal entre os entre vistados éde $R \$ 500,00$ a $R \$ 2.000,00$. Portanto, as políticas de saúde devem abranger o desenvolvimento de programas socioeducativos que orientem a população sobre a importância da saúde bucal para o bem-estar decada pessoa eseja eficiente para atingir a população mais necessitada.

Quanto à faixa etária, observou-semaior frequência dos usuários de 20 a 29 anos em ambos os sexos e a prevalência dos usuários ao serviço por sexo é maior no feminino. Estes resultados são comuns aos estudos de Benitez ${ }^{1}$. Provavelmentecondicionados pela inserção da mulher no mercado formal de trabalho, com maior disponibilidade de tempo, além de uma possível percepção diferenciada do seu processo saúde-doença. Por outro lado, o horário de funcionamento e a oferta de atendimento não facilitam o acesso da população masculina adulta ao serviço².

Os resultados obtidos na coleta dos dados no perfil nosológico apontam que o tratamento mais procurado foi de dentística, ficando claro o perfil de tratamento curativo e não preventivo. Observou-se que os pacientes passam pela disciplina de dentística em grande número, quadro este equivalenteao queacomete o serviço público municipal e estadual, sendo assim necessário ações para aumentar os procedimentos educativos para a prevenção em saúde bucal que deveriam ser mais explorados para tentar reverter 0 quadro do atendimento curativo ${ }^{9}$.

Com relação aos resultados apresentados do perfil das perdas dentais, foi possível observar um quadro alarmante. As razões clínicas para 
esteincluem dor, cárie dental edoença periodontal, tratamento ortodôntico, tratamento pré-protético, trauma eimpactação dental. A minimização dessas razões depende também do critério de diagnóstico, do plano de tratamento adequado e orientação bucal por parte dos dentistas ${ }^{10}$.

A análise dos resultados obtidos dos dados com relação à perda de um ou mais dentes depende de vários fatores; porém, o mais importante salientar éque, dentretodososfatores, uma das principais etiologias da perda dentária é a condição sociocultural e econômica em que se encontra a pessoa.

Já a pressão arterial (PA) é fruto de uma complexa interação de fatores fisiológicos que mantêm e adaptam o ser humano às diversas condições a que é exposto. Considerando que 10 a $20 \%$ dos brasileiros com mais de vinte anos de idade sejam hipertensos e, desses, apenas $40 \%$ estejam cientes do fato, e considerando, ainda, que grande parte da população possui um certo receio em receber tratamento odontológico eque este receio pode alterar a PA, éimportante quese avalie a PA no âmbito do procedimento odontológi co ${ }^{11,12}$. Muitos pacientes assintomáticos, que regularmente vão ao dentista, poderiam tomar consciência da al teração de sua PA através da simples aferição desta ${ }^{13}$.

U mas das patologias relacionadas com a PA é a hipertensão arterial sistêmica (HAS) , que é uma doença de caráter multigênico e multifatorial que pode ser catalogada dentre as doenças crônica degenerativa desdeque, não tratada adequadamente, acarreta danos ao organismo, principalmenteem relação ao coração, rins, e sistema nervoso ${ }^{14}$. A alta incidência de H AS torna necessária a verificação da PA como conduta de rotina em todos os serviços odontológicos, constituindo-se importante medida para se evitar reações adversas em procedimentos odontológicos em pacientes hipertensos.
Os achados desta pesquisa são de extrema importância para melhor planejar as ações de promoção de saúde dentro da nova Policlínica Odontológica da UEA, uma vez que o projeto pedagógico do curso de odontologia enfatiza a valorização do cirurgião-dentista generalista, para que nosso egresso, possa atender as necessidades da população do estado, que possui características peculiares quanto à saúde bucal.

Com os resultados destes trabalhos, os dirigentes da instituição poderão visualizar melhor nosso público-alvo e praticar uma odontologia baseada em evidências ${ }^{15}$, definida como o uso consciente, explícito e criterioso da melhor evidência científica disponível na tomada dedecisões sobre o cuidado de nossa população esuas necessidades, podendo ser aplicado este conceito nos planos de ensinos das disciplinas de semiologia e até nos estágios curriculares realizados na capital e no interior do estado. É importante salientar que, em conjunto com os estudos de demanda ambulatorial, deveriam ser feitos os estudos de satisfação dos usuários, podendo assim acelerar as correções das distorções ocorridas durante 0 processo de atendimento ao usuário.

\section{Considerações finais}

Conclui-se que a população predominantemente atendida durante a realização deste estudo foi de mulheres de classe média baixa, com nível médio de instrução e quadro odontológico precário, evidenciando muitas perdas dentais e ne cessitando mais detratamentos reabilitadores do que os preventivos. A partir do conhecimento das características da demanda ambulatorial da clínica UEA, será possível melhorar o planejamento do atendimento aos usuários e possibilitar uma melhor formação acadêmica social do discente, para que este entre no mercado de trabalho e promova saúde para a população. 


\section{Colaboradores}

EC Sponchiado Júnior eTB Souza participaranm igualmente de todas as etapas da elaboração do artigo.

\section{Agradecimentos}

Agradecemos à Coordenadora do $\mathrm{PAICl}$, Profa. Dra. Hellen Emília de Souza, pelo apoio durante a realização deste trabalho, e a FAPEAM, pelo apoio financeiro a este projeto.

\section{Referências}

1. Benitez G. Morbilidad em consultas ambulatoriales Del Policlínico de la Comunidad "Belkis Sotomayor". Rev Cub Admin Salud 1985; 1(1):234-242.

2. Carvalho M S. Demanda Ambulatorial em três serviços da rede pública do município do Rio de Janeiro. Cad Saude Publica 1994; 10(1):113-115.

3. Frazão $P$, Antunes JLF, Narvai PC. Early tooth loss in adults aged 35-44: State of Sao Paulo, Brazil. 1998. Rev. bras. epidemiol. 2003; 6(1):49-57.

4. Pinto VG. Relacionamento entre padrões de doença e serviços de atenção odontológica. Rev. Saude Publica 1989; 23:509-514.

5. Gomes MA, Pierin M, Angela M G, Segre CA. Home blood pressure measurement and ambulatory blood pressure measurement versus office blood pressure measurement. Arq Bras Cardiol 1998; 71(4):581-585.

6. Pinto VG. Saúde bucal no Brasil. Rev. Saude Publica 1983; 23(5):9-14.

7. Instituto Brasileiro de Geografia e Estatística. Pesquisa nacional por amostra de domicílios. 1999 [CD-ROM ]. M icrodados. Rio de Janeiro: IBGE; 2005.

8. Vargas AMD, Paixão HH. Perda dentária e seu significado na qualidade de vida de adultos usuários de serviço público de saúde bucal do Centro de Saúde Boa Vista, em Belo Horizonte. Cien Saude Colet 2005; 10(4):1015-1024.

9. Marmot $M$. Social determinants of health: from observation to policy. Med J 2000; 172(8):379-382.

10. Silveira CJ, Junior AFC, Souza EHA, Gusmão ES. Razões das perdas dentárias nas cidades de Maceió e Recife, Brasil. Arq em Odontol BH 2004; 40(3):207286.

11. M oreira LB, Fuchs FD, M oraes RS. Prevalence of smoking and associated factors in a metropolitan area of southern Brazil. Rev. Saude Publica 1995; 29(1):46-51.

12. Landim AA. Indicadores de qualidade para os serviços de odontologia pública municipal. M anaus: Universidade do Estado do Amazonas; 2005.

13. Robbins SL. Robbins patologia estrutural e funcional. 6a ed. Rio de Janeiro: Guanabara Koogan; 2000.

14. Piccini RX, Victora CG. Hipertensão arterial sistêmica em área urbana no sul do Brasil: prevalência e fatores de risco. Rev. Saude Publica 1994; 28(4):261267.

15. Sackett D, Richardson W, Rosenberg W, Haynes R. Evidence-based medicine: How to practice and teach EBM . New York: Churchil Livingstone; 1997.

Artigo apresentado em 28/04/2008

Aprovado em 10/10/2008

Versão final apresentada em 29/10/2008 